\title{
Particle Volatility Volatile Particles Measured by Vapor-Particle Separator
}

\author{
Meng-Dawn Cheng ${ }^{1}$ and Edwin Corporan ${ }^{2}$ \\ ${ }^{1}$ Oak Ridge National Laboratory, Environmental Sciences Division, Oak Ridge, TN, U.S.A. \\ ${ }^{2}$ Air Force Research Laboratory, Aerospace Systems Directorate, Wright-Patterson, OH, U.S.A.
}

\begin{abstract}
Vapor-Particle Separator (VPS) is a new technology developed for characterization of the volatile fraction of particulate matter in a combustion aerosol population. VPS incorporates a novel metallic membrane and operates in a cross-flow filtration mode for separation of vapor and solid (i.e. non-volatile) particles. Demonstration of the VPS technology on aircraft engineemitted particles has led to the improvement of the technology and increased confidence on the robustness of its field performance. In this study, the performance of the VPS was evaluated against the Particle Measurement Programme (PMP) volatile particle remover (VPR), a standardized device used in heavy duty diesel engines for separation and characterization of nonvolatile particulate matter. Using tetracontane particles in the laboratory reveals that the VPS performed reasonably well in removing the volatile species. In the field conditions, a singlemode particle size distribution was found for emitted particles from a T63 turboshaft engine at both idle and cruise engine power conditions. Removal of the volatile T63 engine particles by the VPS was consistent with that of PMP VPR. In tests on an F117 turbofan engine, the size distribution at the idle (4\% rated) engine power condition was found to be bimodal, with the first mode consisting of particles smaller than 10nm, which are believed to be mostly semi-volatile particles, while the second mode of larger size was a mixture of semi-volatile and non-volatile particles. The distribution was single modal at the $33 \%$ rated engine power with no secondary mode observed. Overall, for particles emitted by both engines, the removal efficiency of the VPS appears to surpass that of the PMP VPR by $8-10 \%$.
\end{abstract}

This manuscript has been authored by UT-Battelle, LLC under Contract No. DE-ACO5OOOR22725 with the US Department of Energy. The United States Government retains and the publisher, by accepting the article for publication, acknowledges that the United States Government retains a non-exclusive, paid-up, irrevocable, world-wide license to publish or reproduce the published form of this manuscript, or allow others to do so, for United States Government purposes. The Department of Energy will provide public access to these results of federally sponsored research in accordance with the DOE Public Access Plan (http://energy.gov/downloads/doe-public-access-plan). 
Manuscript Prepared for Submission to J Aerosol Science

KEYWORDS: Volatile Particles, Engine Emissions, Vapor-Particle Separator, F117

Engine, T63 Engine 


\section{INTRODUCTION}

Combustion particles from aircraft engines are composed of condensable and non-volatile fractions (Cheng et al., 2009), and all turbine engine particles are ultrafine (Cheng et al., 2008 \& 2009). Small size of particles enhances their ability to translocate if they are inhaled (Oberdörster and Utell, 2002). Numerous epidemiological studies have clearly shown a relationship between particulate matter $(\mathrm{PM})$ concentrations and mortality rates. $\mathrm{PM}_{2.5}$ or particles $\leq 2.5 \mu \mathrm{m}$ in aerodynamic diameter is a criteria pollutant by the U.S. Environmental Protection Agency (EPA). The U.S. EPA and international environmental agencies continue to implement more stringent air quality standards. These standards and regulations will no doubt affect the transportation sector, including aviation industry that relies heavily on the use of fossil fuels. In the case of aviation, the regulation may slow the growth of commercial aviation worldwide and limit operations in U.S. military bases, especially those located within National Ambient Air Quality non-attainment areas.

More than $50 \%$ of the atmospheric PM mass is classified as secondary in many regions such as the Southeast U.S. Secondary PM is a result of the complex gas-to-particle transformation and mass transfer between the two phases. Molecules of higher molecular weight, including sulfur-based and organic compounds, are responsible for the formation of secondary PM (referred to as volatile PM - VPM) in the atmosphere. As the exhaust leaves the engine, it rapidly mixes with the ambient air and cools down, high molecular weight vapor molecules in the exhaust plume would condense onto the non-volatile fraction (or soot) (Ristimaki et al., 2007). Given a sufficient concentration, the vapors could nucleate into new particles through the condensation-nucleation processes (Ronkko et al., 2007) that would change the particle size distribution from a single mode distribution to possibly a bi-modal one of non-equal size. Representative sampling and accurate measurement of the volatile aircraft engine PM has been challenging as these are greatly influenced by the ambient conditions and composition of the engine exhaust. At the present, only soot or soot-like materials or non-volatile PM (primary PM) can be measured consistently in the particulate phase by modern aerosol instrument and sampling technology as demonstrated in the Particle Measurement Programme (PMP) (Giechaskiel et al., 2008; Mamakos, 2012; Zheng et al., 2012).

There exists no standard method for sampling and measurement of VPM. Currently, researchers remove aircraft volatile PM by using devices such as thermodenuder (Burtscher et al., 
2001), catalytic stripping (Swanson and Kittelson, 2010; Mamakos, 2012), and Volatility Tandem Differential Mobility Analyzer (VTDMA) (Johnson et al., 2004; Villani et al., 2007). None of these enable one to observe engine particle dynamics, molecular transfer, or evaporation process under varying temperature conditions. For example, the thermodenuder and the catalytic stripper were intended for rapid removal of volatile components from soot particles, while VTDMA was to investigate volatilization and hygroscopicity of single ambient aerosol particles. Although highly detailed, the mobility analyzer the VTDMA is too slow to be suitable for aircraft VPM measurement. In addition, the concentrations of water vapor, unburned hydrocarbons, and particles in the engine exhaust are so high that the VTDMA would be overwhelmed.

For 30 years, thermodenuder of different variations has been a popular device designed to desorb volatile species for studies of "solid" ambient particles (Johnson et al., 2004; Newman, 1978; Cobourn et al., 1978; Slanina et al., 1978; Sturges and Harrison, 1988; Wehner et al., 2002; Fierz et al., 2007; Park et al., 2008; Huffman et al., 2008; Wu et al., 2009). Thermal stripping devices have also been used on diesel engine soot and aircraft emissions (Burtscher et al., 2001; Maricq et al., 1999; Vaaraslhti et al., 2004; Virtanen et al., 2004; Petzold et al., 2005; Mamakos et al., 2006). Although volatile components are removed from the PM, the adsorbent used in these designs retain the volatile components. Once the adsorption capacity is exceeded, the volatiles could and have been found to re-condense on existing particles or form new ones (e.g., Swanson and Kittelson, 2010).

\section{Description of Vapor Particle Separator (VPS)}

To improve measurement and characterization of aircraft VPM, a new technology called Vapor-Particle Separator (VPS) has been designed and tested at the Oak Ridge National Laboratory (ORNL) (Cheng and Allman, 2011). The VPS technology was tested against the PMP VPR (Giechaskiel et al., 2008; Mamakos, 2012; Zheng et al., 2012) using tetracontane particles and exhaust particles from turbine engines (T63 and F117 engines at the WrightPatterson Air Force Base). The PMP VPR requirements have been modified to accommodate the smaller particles emitted by turbine engines relative to diesel engines. The VPS has met the total volatile particle removal requirements, but additional tests are needed to demonstrate compliance with size-dependent losses of non-volatile particles and other VPR requirements. 
These had not been established at the time the VPS was evaluated.

It is important to mention that the PMP VPR is not a standard for aircraft emission measurement, although it has been used as a standard protocol in diesel engine research and certification (Giechaskiel et al., 2009). Its use and the results reported in this paper are primarily for comparison with those of the VPS. Currently, there isn't a standard VPR for non-volatile PM measurements from aircraft turbine engines. The SAE E31 committee has established requirements for a compliant VPR in the draft of the nvPM Aerospace Recommended Practice (ARP) 6320. The ARP will be balloted by December 2016. If its approved, then the VPR as established in the ARP will be the standard.

In contrast to current commercially available thermodenuders that use adsorption as the means of removing vapor from particles, the VPS technique applies the principle of membrane separation in a counter-flow filtration design to remove vapors and thus prevent re-condensation of desorbed vapor onto existing particles. This approach has been found to be effective in separating vapor from particles at a given temperature, but also permits the collection of vapor (e.g., by canisters, solid-phase extraction cartridges, etc.) driven out from the particles. The collected vapors can be subsequently analyzed chemically if so desired, which is an added capability unavailable in current thermodenuder or catalytic strippers used as volatile particle removers.

Furthermore, operationally, the new design eliminates the need to replace the adsorbents, which greatly simplifies the maintenance of the instrument. In the VPS construction, a microporous metallic membrane effects the separation of vapors and solid particles. The metallic membrane is chemically inert and similar to that used in a previous study of water treatment (Phelps et al., 2008). Many different metallic materials have been used in the construction of the membrane. The membrane made specifically for this project is fabricated as double-layered from 306L stainless steel, and it is about 400 microns thick when two layers are combined. The pore size of the top layer is from 5 to $500 \mathrm{~nm}$ and about 10 microns thick, and is supported by a backbone structure with a pore size of 500 to $50,000 \mathrm{~nm}$ with a thickness less than 400 microns. The VPS design concept was awarded by a U.S. Patent Number US 8,771,402 B2 on July 8, 2014. An early paper (Cheng and Allman, 2011) described in detail the construction of the VPS. 
The VPS instrument was evaluated using synthetic particles generated in an aerosol laboratory at ORNL. These synthetic particles included: (1) a semi-volatile material DOP $\left(\mathrm{C}_{24} \mathrm{H}_{38} \mathrm{O}_{4}\right)$, (2) a non-volatile material $\mathrm{NaCl}$, and (3) a semi-volatile material, tetracontane $\left(\mathrm{C}_{40} \mathrm{H}_{82}\right)$, considered as the test particles simulating aircraft engine emitted particles (Mamakos et al., 2006). Additional information regarding the VPS construction and laboratory tests can be found in the Strategic Environmental Research and Development Program (SERDP) project report (https://www.serdp-estcp.org/Program-Areas/Weapons-Systems-and-Platforms/Noiseand-Emissions/Air-Emissions/WP-1627) and some published journal manuscripts (e.g., Cheng et al., 2009; Cheng, et al., 2008; Cheng and Allman, 2011; Cheng, 2010).

\section{MATERIALS AND METHOD}

Evaluation of the VPS was performed in laboratory and field conditions. The evaluation in the laboratory was designed to (1) understand particle transmission efficiency at non-heated condition (Cheng, 2010; Cheng and Allman, 2011), (2) assess the feasibility for separation of gas phase species vaporized from the particles, and (3) evaluate the removal efficiency of particles as a function of temperature. Early studies (Cheng and Allman, 2011; Cheng, 2010) had addressed the first two to some extent using sodium chloride $(\mathrm{NaCl})$, dioctyl phthalate (DOP, $\mathrm{C}_{24} \mathrm{H}_{38} \mathrm{O}_{4}$, molecular weight $=390.56 \mathrm{~g} \mathrm{~mol}^{-1}$ ), and other inorganic materials. We update the results for (2) above using particles produced by DOP and report new data for (3) using particles generated from tetracontane $\left(\mathrm{C}_{40} \mathrm{H}_{82}\right.$, molecular weight $\left.=563.10 \mathrm{~g} \mathrm{~mol}^{-1}\right)$.

\section{Collection and Detection of Vapors Removed from Particles}

DOP (CAS Number 117-81-7, Bis(2-ethylhexyl) phthalate) was purchased from Sigma Aldrich and used without further purification. A $0.01 \% \mathrm{w}$ DOP solution $(1 \mathrm{~g}$ of DOP material in $99 \mathrm{~g}$ of butanol) was used to generate aerosolized particles by a commercial nebulizer (TSI model 3076). Since one of the unique features of the VPS is its ability to collect vapor molecules through the vapor-particle separation process, it was tested on DOP particles. Vapors collected from the membrane side of the VPS were found to be those vaporized from the DOP particles (Cheng and Allman, 2011; Cheng, 2010). The gas chromatogram indicated (not shown here) the presence of evaporated DOP molecules plus a number of smaller molecules (e.g., octyl 
hydrogen phthalate, phthalic acid, and benzoic acid) resulted from decomposition of the DOP molecules.

However, when vapors were previously collected from the T63 engine (operated at the idle condition) exhaust in a field campaign, the gas chromatogram of the vapors for a VPStemperature of $25^{\circ} \mathrm{C}$ resembled that of the $300^{\circ} \mathrm{C}$, in both chemical components identified and their magnitude(see Figure 1). The vapors analyzed were mostly unburned jet fuel, although there were some tiny peaks that might indicate small molecules decomposed from combustion of the fuels. The overall resultant same magnitude at the two VPS temperatures suggest that the unburned hydrocarbon vapors were removed from the exhaust stream and that the non-volatile particles were reasonably "dry" as no additional components could be observed when the particles were heated to $300^{\circ} \mathrm{C}$. Conversely, using a longer residence time in the VPS might improve the amount of vapor molecules stripped off from the particles; however, this was not pursued because the residence time was $2.6 \mathrm{sec}$ (at a VPS flow rate of 1.5/0.5 aerosol/vapor SLPM). Increasing the residence time would require decreasing the flow rate that could disrupt the cross-flow VPS separation, something we decided not to attempt during the field test.

It was however clear from the earlier T63 campaign that detection of semi-volatile species removed from VPM in aircraft engine particles by using VPS is much more challenging than previously thought. Again, this is because the vapors from unburned jet fuel would overwhelm the signal as we observed. Although the VPS's utility for simultaneous vapor and particle collection is unique, it appears that at the present the ability is best suited for as a sorbent-free vapor particle separator to minimize the possibility of vapor recondensation (Burtscher et al., 2001; Swanson and Kittelson, 2010). This vapor-particle separation ability prolongs the duty cycle of the VPS in comparison to that of a thermodenuder that requires sorbent for vapor removal.

\section{Evaluation of VPS Particle Removal by Using Tetracontane}

Tetracontane $\left(\mathrm{C}_{40} \mathrm{H}_{82}\right)$ (called $\mathrm{C}_{40}$ hereafter) is the standard material used for the PMP VPR performance validation. $\mathrm{C}_{40}$ material is basically a 40 -carbon alkane and considered non-volatile at room temperature $\left(25^{\circ} \mathrm{C}\right)$ based on its vapor pressure $\left(\sim 5.4723 \times 10^{-13} \mathrm{~Pa}\right)$ (Chickos et al., 2008). The $\mathrm{C}_{40}$ material (CAS \# 4181-95-7) was purchased from Sigma Aldrich and used as received. The $\mathrm{C}_{40}$ test particles were generated by using the evaporation-condensation technique (See 
Figure 2a for setup). Operationally, the pre-weighted $\mathrm{C}_{40}$ powder was placed in a quartz boat that was inserted into a stainless steel tube of 1" ID inside the Lindberg linear furnace (to the left side of the figure). The furnace was operated at a constant temperature of $160^{\circ} \mathrm{C}$. A 0.7 SLPM particle-free air flow into the stainless steel tube sweeping the evaporated $\mathrm{C}_{40}$ molecules. As this $\mathrm{C}_{40}$ vapor entering the stainless steel manifold, a 1.3 SLPM particle-free air was added to the 0.7 SLPM airflow at the point labeled A in Figure 2a to make a total of 2.0 LPM flow. The additional air was at the a temperature of $21^{\circ} \mathrm{C}$; it cooled the hot vapor stream and condensed $\mathrm{C}_{40}$ molecules to form particles comprised of $\mathrm{C}_{40}$ molecules.

The $\mathrm{C}_{40}$ particles were sampled in the cool zone of the generator (region labeled B Figure 2a) using a $1 / 4 "$ retractable sampling tube. The extracted sample was sent to various measurements including the VPS, Raman spectroscopy and an online continuous aerosol concentration monitor. Figure $\mathbf{2} \mathbf{b}$ shows the Raman spectrum of the collected $\mathrm{C}_{40}$ particles taken at the room temperature of $21^{\circ} \mathrm{C}$. The characteristic double peak C-H stretch band around 2800$3000 \mathrm{~cm}^{-1}$ clearly identifies the $\mathrm{C}_{40}$ molecules. The smaller peaks in the $1400-1500 \mathrm{~cm}^{-1}$ region correspond to the $\mathrm{H}-\mathrm{C}-\mathrm{H}$ stretch. The spectrum was reasonably clean indicating the purity of the $\mathrm{C}_{40}$ particles generated for the test.

The generated aerosol stream was monitored for concentration stability using a TSI 3068B aerosol electrometer. The particles were charged by a Kr-85 neutralizer before they entered the electrometer. The averaging time for each data point was ten seconds. Figure $2 \mathbf{c}$ shows an example of time trace of the measured $\mathrm{C}_{40}$ aerosol particles. The number concentration fluctuated some at the start up then it stabilized at about $10^{5}$ particles per $\mathrm{cm}^{-3}$. When the concentration stabilized it was the time the VPS experiment started. The tetracontane source was depleted after approximately 3.8 hours and the concentration decreased toward the end (see Figure 2c). The VPS experiment ended before the particle concentration decreased to less than $100 \mathrm{~cm}^{-3}$.

The Organic Carbon (OC) and Elemental Carbon (EC) measurement follows the NIOSH 5040 method for diesel particulate (http://www.cdc.gov/niosh/docs/2003-154/pdfs/5040.pdf) utilizing a Sunset Carbon Analyzer running thermal-optical detection on collected quartz particulate filters (Birch and Cary, 1996). Quartz filters were pre-fired in a nitrogen-muffed furnace overnight and placed individually in a plastic cartridge. The minimal quantifiable levels 
of detection are approximately $0.4 \mu \mathrm{g} \mathrm{m}^{-3}$ of OC and EC. The collection time for engine exhausts ran approximately 5 minutes at a 23 SLPM flow rate.

\section{RESULTS AND DISCUSSION}

\section{Thermal Behavior of $C_{40}$ Aerosol Particles}

Thermal degradation of tetracontane was previously studied (Németh et al., 2008) in many applications including waste treatment and polymer manufacturing. Reactions at high temperature (e.g., $500^{\circ} \mathrm{C}$ ) were found to involve simultaneous or subsequent $\mathrm{H}$-abstraction, $\beta$ scission and backbiting reactions (intramolecular H-shifts) leading to the formation of radicals, smaller alkane, alkene, diene molecules, and terminally unsaturated polymer residues (Németh et al., 2008). Some or all of these reactions might still exist in our study even the $\mathrm{C}_{40}$ material was present in an aerosol form instead of a bulk polymer material. As discussed earlier, we focus this report on the particle removal capability of VPS. No collection of vapor phase material was conducted for this study, but it is a subject for future study.

The legend "Source" in Figure 3 denotes the $\mathrm{C}_{40}$ particles generated by the evaporationcondensation technique discussed earlier. The $\mathrm{C}_{40}$ particles were size-selected by a differential mobility analyzer (DMA) to form a population of test particles (geometric mean diameter $=$ $89.2 \mathrm{~nm}$ ) of practically one size. The geometric standard deviation for the "Source" peak was 1.04, which indicates the 89-nm particle population was practically monodisperse. The 89-nm $\mathrm{C}_{40}$ particles were heated at four different temperatures $\left(50,75,100\right.$, and $\left.350^{\circ} \mathrm{C}\right)$ in this study. The population of the $\mathrm{C}_{40}$ particles in a size distribution curve for each of the four temperatures is displayed in Figure 3. As shown, the peak of the size distributions of the $\mathrm{C}_{40}$ particles leftshifted as the temperature was increased. The geometric mean diameter was around $40 \mathrm{~nm}$ at $50^{\circ} \mathrm{C}$, and 33 at 75,28 at $100^{\circ} \mathrm{C}$. The decrease of the peak particle diameter as temperature decreased was approximately log-linear. One also noticed that all the $89-\mathrm{nm} \mathrm{C}_{40}$ particles were removed at an efficiency greater than $99.99 \%$ as the VPS temperature went up to $350^{\circ} \mathrm{C}$. A similar experimental setup to that shown in Figure 2a was performed by selecting the $\mathrm{C}_{40}$ particles smaller than $15-\mathrm{nm}$; however, it did not produce a meaningful result because the number concentration of the $\mathrm{C}_{40}$ particles at the source was too low. It is highly likely that the removal of $15-\mathrm{nm}$ and smaller $\mathrm{C}_{40}$ particles would be greater than $99.99 \%$ at $350^{\circ} \mathrm{C}$ as well. 
It is important to note that the laboratory evaluate of VPS could only provide feasibility data supporting our design and efficiency assessment under an ideal condition. Only the field performance of the VPS instrument can indicate its effectiveness of being a volatile particle remover, which is the topic of the following Sections.

\section{Evaluation Using Aircraft Engine-Generated Particles}

The PMP Working Group established a protocol for measuring non-volatile PM from light and heavy duty vehicle engines (Giechaskiel et al., $2008 \& 2009$ ). This included a volatile particle remover (VPR) for removing volatile species. Although this PMP is not an accepted protocol for aircraft particulate emissions; it was used here simply as a comparison to the VPS particle removal capability of aircraft engine-generated volatile PM, not as a reference standard or regulatory authority one may imply.

The ORNL PMP VPR system consisted of a two-stage micro-dilution system designed to vaporize and remove the volatile particles from the engine exhaust, leaving only the solids (nonvolatile) to be measured by the SMPS. The first-stage and second stage dilution ratios are 5:1 and 6:1 respectively, producing an overall dilution ratio of approximately 30:1. The ORNL system is somewhat similar to the commercial PMP VPR system; however, it uses a lower dilution ratio to provide a greater number of particles for statistically significant SMPS numbersize distributions. ORNL modified the PMP sampling system to allow for quantitation of particles as small as $10 \mathrm{~nm}$ (versus $23 \mathrm{~nm}$ for the standard PMP protocol), which requires a CPC with a smaller diameter cutoff point such as that of a TSI 3025A Ultrafine Condensation Particle Counter (UCPC). Figure 4 shows a photo of the PMP VPR unit.

\section{Demonstration using T63 Turboshaft Engine}

The T63 engine is a military version of the Allison engine model 250. This engine provides the opportunity to rapidly vary fuel chemical composition (e.g., sulfur and aromatic content) to influence the concentration of volatile PM precursors. A standard JP-8 fuel as well as a blend of the jet fuel with aromatic solvents to increase the aromatic content to the specification maximum of 25\% by volume, were tested in this effort. The T63 engine, shown in Figure 5, is located in the Engine Environment Research Facility (EERF) in the Aerospace System Directorate at 
Wright-Patterson Air Force Base (WPAFB). Note that the fuel used in this test was a standard JP-8 fuel with no modification of its composition.

Figure 6 shows the lognormal fit of the averaged size distribution for the particles measured by using either VPS or PMP VPR under different engine power conditions. Figures $\mathbf{6 a}$ and $\mathbf{6 b}$ are the lognormal-fitted particle size distribution curves for the PMP VPR-conditioned sample, while $\mathbf{6 c}$ and $\mathbf{6 d}$ are for the sample after the VPS. The mode diameters were $38 \mathrm{~nm}$ for the engine idle (6a) power condition and $62 \mathrm{~nm}$ for the cruise power (6eb) condition by the PMP method. The mode diameter of the distributions at the cruise condition was slightly reduced (see Figure 6b) as the temperature was increased from $150^{\circ} \mathrm{C}$ to $350^{\circ} \mathrm{C}$. No $350^{\circ} \mathrm{C}$-data were obtained by PMP VPR for the idle power condition. The mode diameters of both VPS-conditioned curves remained constant as the temperature increased. Also note that the mode diameters of VPS and PMP VPR are identical in both engine power conditions. With regards to the removal of particles, both VPS and PMP VPR were able to show that the number concentration decreased as the VPS or VPR temperature increased. At the cruise condition, the reduction in number concentration was slightly higher by VPS than by PMP VPR. In short, measurements by VPS and PMP VPR in terms of particle size distribution statistics (the diameter and peak height) are comparable.

Lognormally fitted particle size distributions for the aromatics-doped fuel blend are shown in Figures 7a-7d. Enhancing the aromatic concentration to the base fuel JP-8 was an experimental attempt to increase the volatile particles generated by the engine combustion. It was interesting to note that the mode diameters in Figure 7 remained close to those without the aromatic additive (see Figure 6). This indicates that the added aromatics did not contribute to the formation of a second particle population. The mode diameters of the particles were reduced slightly by the PMP VPR (Figures 7a and 7b) when the samples were heated to $350^{\circ} \mathrm{C}$. The number concentrations did not change appreciably at the idle engine power condition. The reductions in the peak particle number concentration of-by the VPS particle distributions at $400 \mathrm{C}$ the both idle and cruise power conditions (Figure 7c and 7d) were more pronounced larger than those by PMP VPR as shown in Figures $7 \mathbf{a}$ and $7 \mathbf{b}$. We think these might be caused by the longer residence time ( 3 secs) than that used in the PMP VPR ( 0.3 seconds) in the campaign.

Figure 8 shows the elemental carbon (EC) and organic carbon (OC) on the raw T63 particulate samples and using $\mathrm{OC}$ as the surrogate measure of the volatile component. These 
were measured using the Sunset Analyzer using the NIOSH 5040 methodology. As anticipated, the addition of the fuel aromatics significantly enhanced the production of EC relative to the baseline fuel, however, it had minimal impact on OC.

\section{Demonstration using F117 Turbofan Engine at WPAFB, OH}

The second test was conducted on a C-17 aircraft (F117-PW-100 turbofan engines) in 2015. The F117 engine is the military version of the Pratt \& Whitney PW2000 commercial engine, which powers the Boeing 757-200 aircraft. Figure 9 shows a photo of the sampling rig behind a C-17 engine nozzle. The tests were conducted at Wright-Patterson AFB. Exhaust samples were collected at the engine exit plane and transported to the instruments via heated (160C) lines. The sample was diluted prior to entering the VPS or PMP VPR.

Figure 10 shows three particle size distributions reduced using the data obtained by TSI SMPS $^{\circledR}$ during the field test when the C-17 aircraft was operating at the idle rated engine power condition. The curves are the lognormal-fit of the averaged SMPS data (either the raw engine data, VPS- or PMP VPR-conditioned data). The figure of merit $\left(\mathrm{R}^{2}\right)$ of the lognormal curve fit to the averaged particle data was $0.72,0.95,0.93$, respectively, for the raw sample, VPS, and PMP VPR data representing a moderate (the raw data) and good fit. The error bar on each curve represents the range of data from individual scans. The coefficients of variance (i.e., $\mathrm{COV}=$ range/average in $\%$ ) of the data were $51 \%, 31 \%$, and $26 \%$ from $9-330 \mathrm{~nm}$ for the raw data, VPS, and PMP VPR data, respectively. Since we are comparing the overall particle removal performance of VPS to PMP VPR, only the output from the second stage of the PMP VPR is shown in Figure 10.

In the raw sample, there was an increase in the smaller particle portion of the raw-data distribution (the plot of markers), that is indicative of a large number of particles in the 10nm or smaller size range present. This observation was present in all repeated scans of the raw sample in the campaign. If the increase was indeed caused by the volatile particles, the mode diameter of these particles (the invisible second mode of the distribution in Figure 10) was smaller than 10nm. We did not observe such an increase of particles at such a small size range in the T63 engine (see Figure 6). Since a lognormal fit analysis to the particle size distribution used a single mode assumption, it was unable to fit both the primary engine particles and the volatile

particle population in Figure 10. This could have caused the lower $\mathrm{R}^{2}$ value for the raw sample 
compared to those two for the VPS and PMP VPR data, although larger data variation for the raw samples could contribute to the lower fit, also. Although one could attempt to fit the data with a bimodal lognormal distribution, it was considered the data were insufficient to justify such action.

Significant fraction of particles smaller than $11 \mathrm{~nm}$ was found in a diesel emission study (Zheng et al., 2012). This sub-11-nm fraction of particles decreased as the PMP system's dilution ratio increased from 300 to 1500, but remained unchanged when the PMP temperature increased from 300 to $500^{\circ} \mathrm{C}$. The authors suggested that "incomplete evaporation of semivolatile particles did not contribute to those sub-11-nm particles". Instead, they suggested the particles were formed through re-nucleation of semi-volatile precursors in the exhaust. Although we could not vary the dilution ratio on the VPS operation during the campaign, we observed more than $99 \%$ of these were removed as the temperature increased to $350^{\circ} \mathrm{C}$. This suggests that particles smaller than 10-nm in Figure 10 were semi-volatile engine particles.

Figure 10 shows the reduction of particle concentration using the PMP VPR and the VPS relative to the raw sample. These data support the objective that VPS is volatile particle remover as effective as, if not better than, the PMP VPR. We calculated particle removal efficiency (in \%) by taking the difference of the total particle count from the VPS or PMP VPR to the raw sample divided by that of the raw sample and multiplying by 100 to make a percentage value. The particle removal efficiency varied slightly as a function of particle size, but overall for the idle (or 4\%)-rated power condition the particle removal efficiency by VPS is estimated to be $82.3 \%$, and that for PMP VPR is $75.5 \%$.

It may be arguable that larger (say 300nm) particles in the size spectrum are non-volatile, but that may not be true for particles from actual turbine engines as these larger particles might still contain volatile components that could be vaporized by PMP VPR and VPS. The dynamics of particle size change as result of the thermal separation of the non-volatile particles and volatile products in the PMP VPR and VPS makes it difficult to support that all larger turbine engine particles are non-volatile and should not be removed. The best way to verify the argument that larger particles are all non-volatile and cannot be vaporized at $350^{\circ} \mathrm{C}$ is to perform the tandem DMA analysis like we did in the tetracontane case. However, this was not possible in a field campaign like ours. 
Figure 11 shows the particle size distributions of the lognormal fit using data from the C-17 engine emission when the engine was operating at a $33 \%$ rated power. Also included as an error bar are the ranges of the particle measurement data for each individual curve. The particle number concentration of the incoming raw sample was about an order of magnitude higher than that of the idle condition. The COV was $26 \%, 21 \%$, and $15 \%$, respectively, for the raw sample, VPS, and PMP VPR data. The $\mathrm{R}^{2}$ value was $0.83,0.94$, and 0.95 for the lognormal fit to the raw sample, VPS, and PMP VPR data, respectively. It is important to note that particles below 10$\mathrm{nm}$ in diameter were not observed for this condition, and the lognormal fit has a better R2 indicating an improved fit. This was anticipated as volatile PM usually exist at the lower engine power settings where combustion efficiencies are lower and organic species from unburned fuel are higher. However, as shown, even at this higher power setting, significant engine particles were removed both by the PMP VPR and VPS. The overall removal efficiency was calculated to be about $88 \%$ for VPS vs. $80 \%$ for PMP VPR.

\section{CONCLUSIONS}

The Vapor-Particle Separator (VPS) is a new technology for analyzing aerosol volatility. The VPS was designed to separate volatile and non-volatile particles to enhance the characterization of both fractions by using a novel metallic membrane and cross-flow separation. The VPS was field-tested on raw PM samples from military aircraft engines. The utility as a particle remover was compared with that of a PMP VPR device in this study. It was learned from both field (actual engine) and laboratory tests that both PMP VPR and VPS devices are capable of removing particles with a high efficiency based on data. The advantages of the VPS technology are:

1. High particle transmission efficiency,

2. No artificial bias in the vapor-particle separation,

3. Enabling simultaneous measurement of vapor and particulate content,

4. Easy maintenance and no consumables; (e.g., sorbent),

5. Field robust and light-weight construction. 
Overall, the field tests indicate that the VPS instrument is robust and can provide additional scientific information regarding the volatility of aircraft engine-emitted particles. Tests of the VPS instruments with atmospheric particles are ongoing.

\section{ACKNOWLEDGEMENTS}

The design and development of VPS was originally supported by SERDP under Project \#WP-1627 in the Weapons Systems and Platforms Thrust Area. This demonstration research was financially supported by the ESTCP administered by the AFRL under ESTCP Project \# WP201317 and DOE Contract Number 2340-V672-13. Oak Ridge National Laboratory is managed by UT-Battelle, LLC, for the U.S. Department of Energy under contract number DE-AC0500OR22725. Dr. John M. E. Storey (ORNL) was instrumental in the assembly and operation of the PMP VPR device used in both T63 and C-17 (F117) campaigns. Dr. Teresa Barone, Mr. Steve Allman, Dr. Brian Bischoff, Dr. Shannon Mahurin, and Dr. Erik Kabela (all ORNL from several research divisions) provided unparalleled technical laboratory and field supports during the research and development throughout this research. The authors sincerely appreciate Dr. Matthew DeWitt, Mr. Christopher Klingshirn, and Mr. Joe Mantz (all UDRI), and the personnel at WPAFB who operated the engines used during the demonstration.

\section{DISCLAIMERS}

Mention of the commercial instruments, model numbers, trade names, chemicals and chemical manufacturers do not represent the endorsement of the authors nor the organizations the authors are associated with.

\section{REFERENCES}

Birch M.E. and R.A. Cary (1996) Elemental carbon-based method for monitoring occupational exposures to particulate diesel exhaust, Aerosol Science and Technology, 25:221-241.

Burtscher, H., U. Baltensperger, N. Bukowiecki, P. Cohn, C. Huglin, M. Mohr, U. Matter, S. Nyeki, V. Schmatloch, N. Streit, and E. Weingartner, Separation of Volatile and Non-Volatile Aerosol Fractions by Thermodesorption: Instrumental Development and Applications, Journal of Aerosol Science 32, 427-442 (2001). 
Cheng, M. -D., E. Corporan, M. J. DeWitt, and B. Landgraf (2009) Emissions of Volatile Particulate Components from Turboshaft Engines running JP-8 and Fischer-Tropsch Fuels, Journal of Aerosol and Air Quality Research 9, 237-256.

Cheng, M.-D., E. Corporan, M. J. DeWitt, C. W. Spicer, M. W. Holdren, K. Cowen, B. D. Harris, R. Shores, R. Hashmonay, and R. Kagann (2008) Probing Emissions of Military Cargo Aircraft: Description of a Joint Field Measurement Program, Journal of Air and Waste Management Association 58, 787-796.

Cheng, M.-D. (2010) Development and Application of a Membrane-Based Thermodenuder for Measurement of Volatile Particles Emitted by a Jet Turbine Engine, Proceedings ASME Gas Turbine Congress and Exposition, Glasgow, Scotland, June.

Cheng, M.-D. and S. L. Allman (2011) Improved Measurement for Volatile Particles: VPS

Design and Laboratory Tests, Review of Scientific Instruments 82(Dec):125106.1-125106.8, DOI: $10.1063 / 1.3665095$

Chickos, J., T. Wang and E. Sharma (2008) Hypothetical Thermodynamic Properties: Vapor Pressure and Vaporization Enthalpies of the Even n-Alkanes from C40 to C76 at T = 298.15 K by Correlation-Gas Chromatography. Are the Vaporization Enthalpies a Linear Function of Carbon Number? Journal of Chemical Engineering Data (53): 481-491.

Cobourn, W. G., R. B. Husar, and J. D. Husar (1978) Continuous In Situ Monitoring of Ambient Particulate Sulfur Using Flame Photometry and Thermal Analysis, Atmospheric Environment, 12, 89-98.

Fierz, M., M. G. C. Vernooij, and H. Burtscher (2007) An Improved Low-Flow Thermodenuder, Journal of Aerosol Science 38, 1163-1168.

Giechaskiel, B., P. Dilara, E. Sandbach, and J. Anderson (2008) Particle Measurement Programme (PMP) Light-Duty Inter-Laboratory Exercise: Comparison of Different Particle Number Measurement Systems, Measurement Science and Technology, 19: 095401 (16pp)

Giechaskiel, B., Carriero, M., Martini, G., and Andersson, J., "Heavy Duty Particle Measurement Programme (PMP): Exploratory Work for the Definition of the Test Protocol," SAE International Journal of Engines 2(1):1528-1546, 2009, doi:10.4271/2009-01-1767.

Huffman, J. A., P. J. Ziemann, J. T. Jayne, D. R. Worsnop, and J. L. Jimenez (2008) Development and Characterization of a Fast-Stepping/Scanning Thermodenuder for Chemically-Resolved Aerosol Volatility Measurements, Aerosol Science and Technology, 42, 395-407.

Johnson, G. R., Z. Ristovski, and L. Morawska (2004) Method for Measuring the Hygroscopic Behavior of Lower Volatility Fractions in an Internally Mixed Aerosol, Journal of Aerosol Science 35, 443-455.

Mamakos, A., L. Ntziachristos, and Z. Samaras (2006) Evaluation of the Dekati Mass Monitor for the Measurement of Exhaust Particle Mass Emissions, Environmental Science and Technology, 40, 4739-4745.

Mamakos, A. (2012) Particle Measurement Programme. Volatile Particle Remover Calibration Round Robin. Final Report. EMRP. JRC Scientific and Policy Reports.

Maricq, M. M., R. E. Chase, D. H. Podsiadlik, and R. Vogt (1999) Vehicle Exhaust Particle Size Distributions: A Comparison of Tailpipe and Dilution Tunnel Measurements, SAE Technical Paper Series, 1999-01-1461.

Newman, L. (1978) Techniques for Determining the Chemical Composition of Aerosol Sulfur Compounds, Atmospheric Environment, 12, 113-125.

Németh, A., Marianne Blazsó, Péter Baranyai, Tamás Vidóczy (2008) Thermal degradation of polyethylene modeled on tetracontane, Journal of Analytical and Applied Pyrolysis 81 (2008) 
$237-242$

Oberdörster, G. and M. J. Utell (2002) Ultrafine Particles in the Urban Air: To the Respiratory Tract — and Beyond, Environmental Health Perspectives, 110, A440-A441.

Park, D., S. Kim, N. K. Choi, and J. Hwang (2008) Development and Performance Test of a Thermo-Denuder for Separation of Volatile Matter from Submicron Aerosol Particles, Journal of Aerosol Science, 39, 1099-1108.

Petzold, A., M. Fiebig, L. Fritzsche, C. Stein, U. Schumann, C. W. Wilson, C. D. Hurley, F. Arnold, E. Katragkou, U. Baltensperger, M. Gysel, S. Nyeki, R. Hitzenberger, H.-R. Giebl, D. J. Hughes, R. Kurtenbach, P. Wiesen, P. Madden, H. Puxbaum, S. Vrchoticky, and C. Wahl (2005) Particle emissions from aircraft engines - a survey of the European project PartEmis, Meteorologische Zeitschrift 14, 465-476.

Phelps, T. J., A. V. Palumbo, B. L. Bischoff, C. J. Miller, L. A. Fagan, M. S. McNeilly, and R. R. Judkins (2008) Micron-pore-size Metallic Filter Tube Membranes for Filtration of Particulates and Water Purification, Journal of Microbial Methods 74, 10-16.

Ristimaki, J., K. Vaaraslahti, M. Lappi, and J. Keskinen (2007) Hydrocarbon Condensation in Heavy-Duty Diesel Exhaust, Environmental Science and Technology, 41, 6397-6402.

Ronkko, T., A. Virtanen, J. Kannosto, J. Keskinen, M. Lappi, and L. Pirjola (2007) Nucleation Mode Particles with a Nonvolatile Core in the Exhaust of a Heavy Duty Diesel Vehicle, Environmental Science and Technology, 41, 6384-6389.

Slanina, J., M. P. Keuken, and C. A. M. Schoonebeek (1987) Determination of Sulfur Dioxide in Ambient Air by A Computer-Controlled Thermodenuder System, Analytical Chemistry, 59, 2764-2766.

Sturges, W. T., and R. M. Harrison (1988) Thermal Speciation of Atmospheric Nitrate and Chloride: A Critical Evaluation, Environment Science and Technology, 22, 1305-1311.

Swanson, J. and D. Kittelson (2010) Evaluation of thermal denuder and catalytic stripper methods for solid particle measurements, Journal of Aerosol Science, 41: 1113-1122.

Vaaraslhti, K., A. Virtanen, J. Ristimaki, and J. Keskinen (2004) Nucleation Mode Formation in Heavy-Duty Diesel Exhaust with and without a Particulate Filter, Environmental Science and Technology, 38, 4884-4890.

Virtanen, A. K. K., J. M. Ristimaki, K. Vaaraslhti, and J. Keskinen (2004) Effect of Engine Load on Diesel Soot Particles, Environmental Science and Technology, 38, 2551-2556.

Villani, P., D. Picard, N. Marchand, and P. Laj (2007) Design and Validation of a 6-Volatility Tandem Differential Mobility Analyzer (VTDMA), Aerosol Science and Technol. 41, 898906.

Wehner, B., S. Philippin, and A. Wiedensohler (2002) Design and Calibration of a Thermodenuder with An Improved Heating Unit to Measure the Size-Dependent Volatile Fraction of Aerosol Particles, Journal of Aerosol Science, 33, 1087-1093.

Wu, Z., L. Poulain, B. Wehner, A. Wiedensohler, and H. Herrmann (2009) Characterization of the Volatile Fraction of Laboratory-Generated Aerosol Particles by Thermodenuder-Aerosol Mass Spectrometer Coupling Experiments, Journal of Aerosol Science, 40, 603-612.

Zheng, Z., T.D. Durbin, G. Karavalakis, K.C. Johnson, A. Chaudhary, D.R. Cocker III, J.D. Herner, W.H. Robertson, T. Huai, A. Ayala, D.B. Kittelson, and H.J. Jung (2012) Nature of Sub-23-nm Particles Downstream of the European Particle Measurement Programme (PMP)Compliant System: A Real-Time Data Perspective, Aerosol Science and Technol., 46, 886896. 


\section{CAPTION OF FIGURES}

\begin{tabular}{|l|l|}
\hline Figure 1 & $\begin{array}{l}\text { Gas chromatograms of T63-engine exhaust particles sampled by VPS } \\
\text { using the vapor port }\end{array}$ \\
\hline Figure 2 & $\begin{array}{l}\text { [a] Schematics for laboratory setup for testing VPS on C40 particles, [b] } \\
\text { MicroRaman spectrum of C } 40 \text { particles, [c] Time trace of particle } \\
\text { number concentration }\end{array}$ \\
\hline Figure 3 & $\begin{array}{l}\text { Particle size distributions of C40 particles plotted as a function of particle } \\
\text { size and temperature }\end{array}$ \\
\hline Figure 4 & $\begin{array}{l}\text { [a] ORNL PMP VPR tested on C-17 aircraft [b] ORNL VPS version 3 } \\
\text { tested on C-17 aircraft }\end{array}$ \\
\hline Figure 5 & $\begin{array}{l}\text { Schematic of field test on T63 engine installed on a test stand at WP } \\
\text { AFRL }\end{array}$ \\
\hline Figure 6 & $\begin{array}{l}\text { Lognormally fitted particle size distributions for JP8 fuel obtained by: } \\
\text { [a] PMP VPR under T63 idle condition, [b] PMP VPR under T63 cruise } \\
\text { condition, [c] VPS under T63 idle condition, and [d] VPS under T63 } \\
\text { cruise condition }\end{array}$ \\
\hline Figure 7 & $\begin{array}{l}\text { Lognormally fitted particle size distributions for JP8+High Aromatic } \\
\text { fuel obtained by: [a] PMP VPR under T63 idle condition, [b] PMP VPR } \\
\text { under T63 cruise condition, [c] VPS under T63 idle condition, and [d] } \\
\text { VPS under T63 cruise condition }\end{array}$ \\
\hline Figure 11 & $\begin{array}{l}\text { Lognormally-fitted particle size distributions for emissions from the C- } \\
17 \text { engine operated at 33\% rated thrust. Error bars represent the ranges } \\
\text { of particle counts of the repeated scans. }\end{array}$ \\
\hline Figure 8 & $\begin{array}{l}\text { EC and OC contents of measured T63 samples } \\
\text { particle counts of the repeated scans. }\end{array}$ \\
\hline Figure 9 10 & $\begin{array}{l}\text { Sampled PW F117 engine on a C-17 aircraft parked at WPAFB } \\
\text { Lognally-fitted particle size distributions for emissions from the C- }\end{array}$ \\
\hline
\end{tabular}




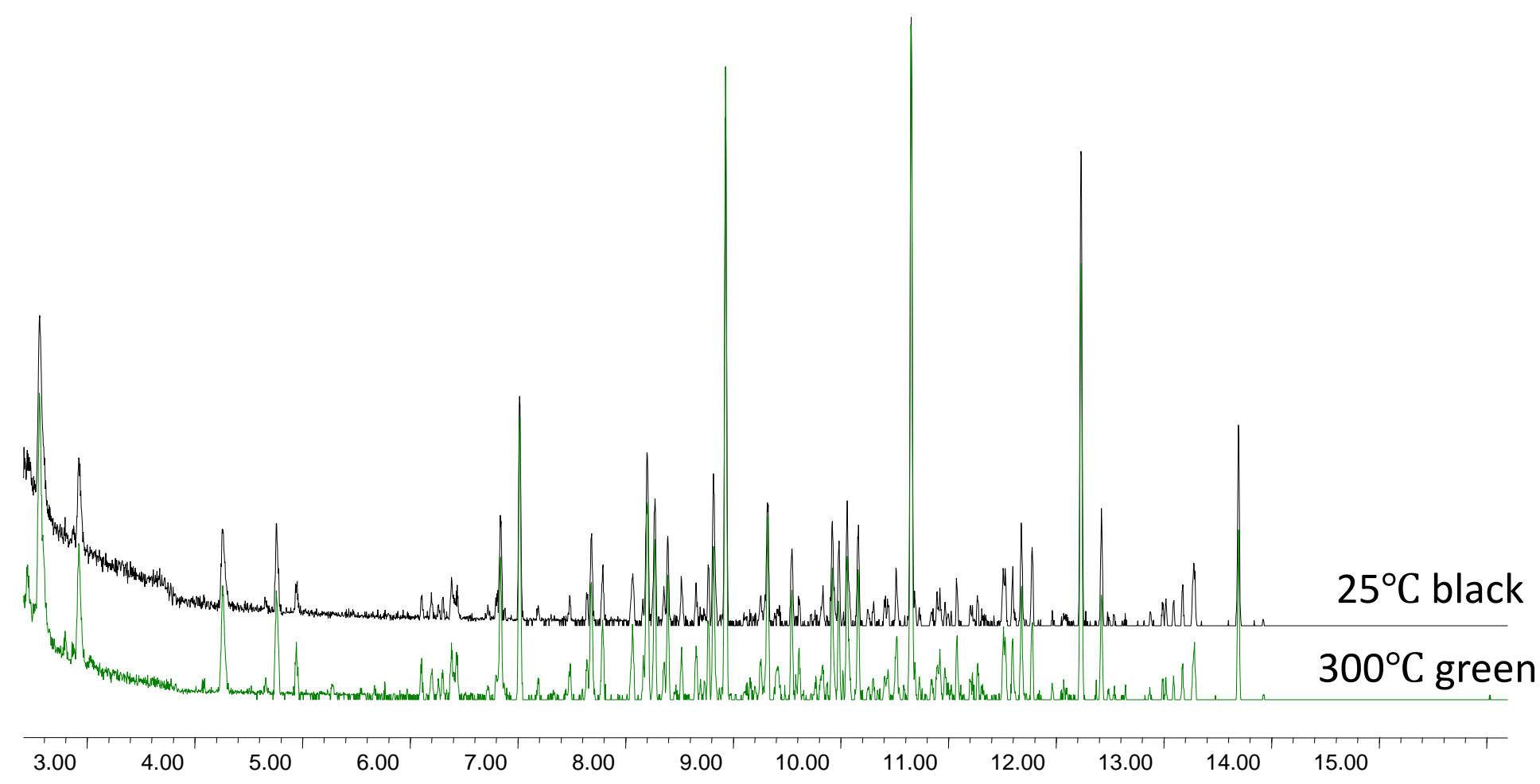

Figure 1. Gas chromatograms of T63-engine exhaust particles sampled by VPS using the vapor port 


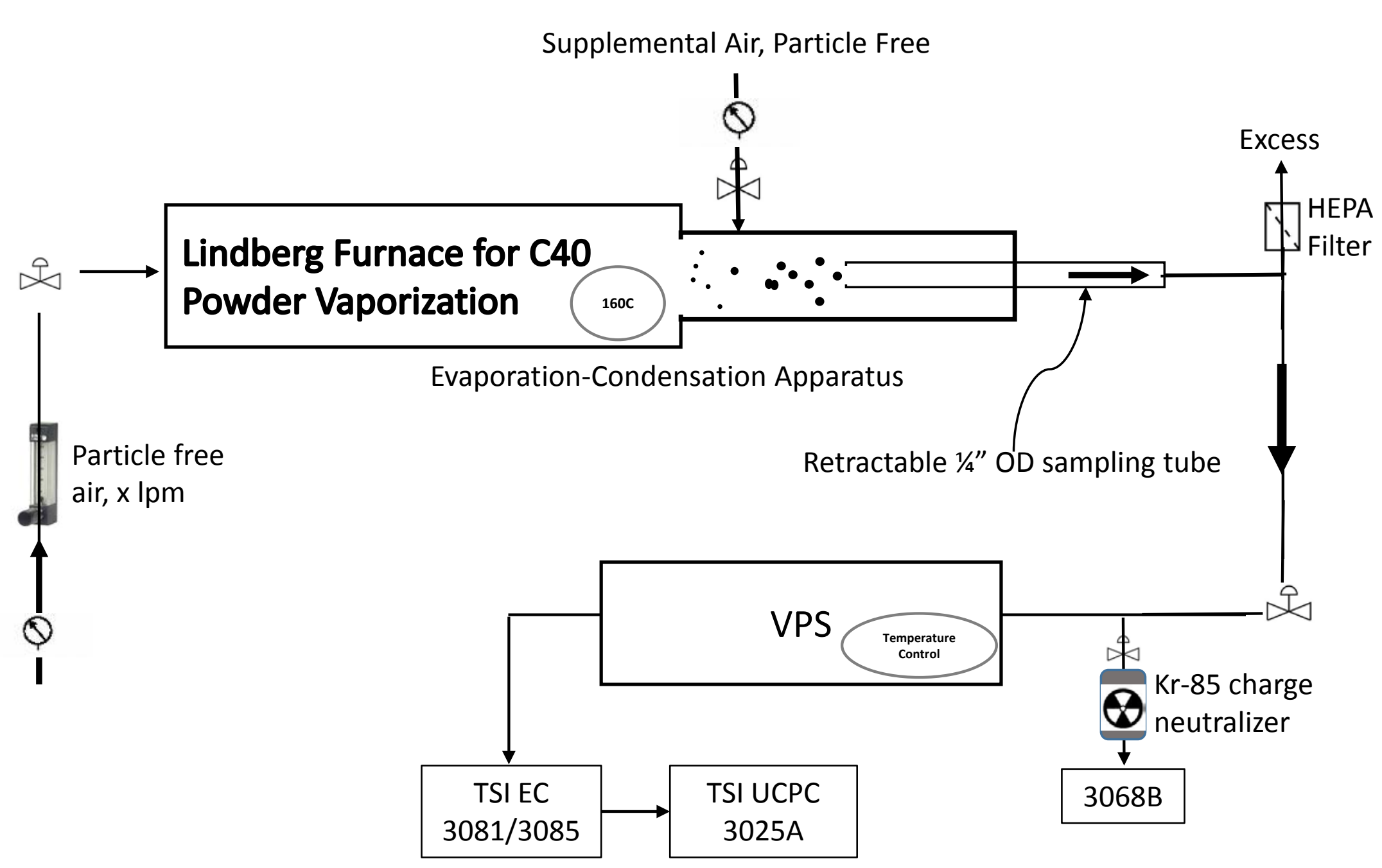

Figure 2a. Schematics for laboratory setup for testing VPS on C40 particles 


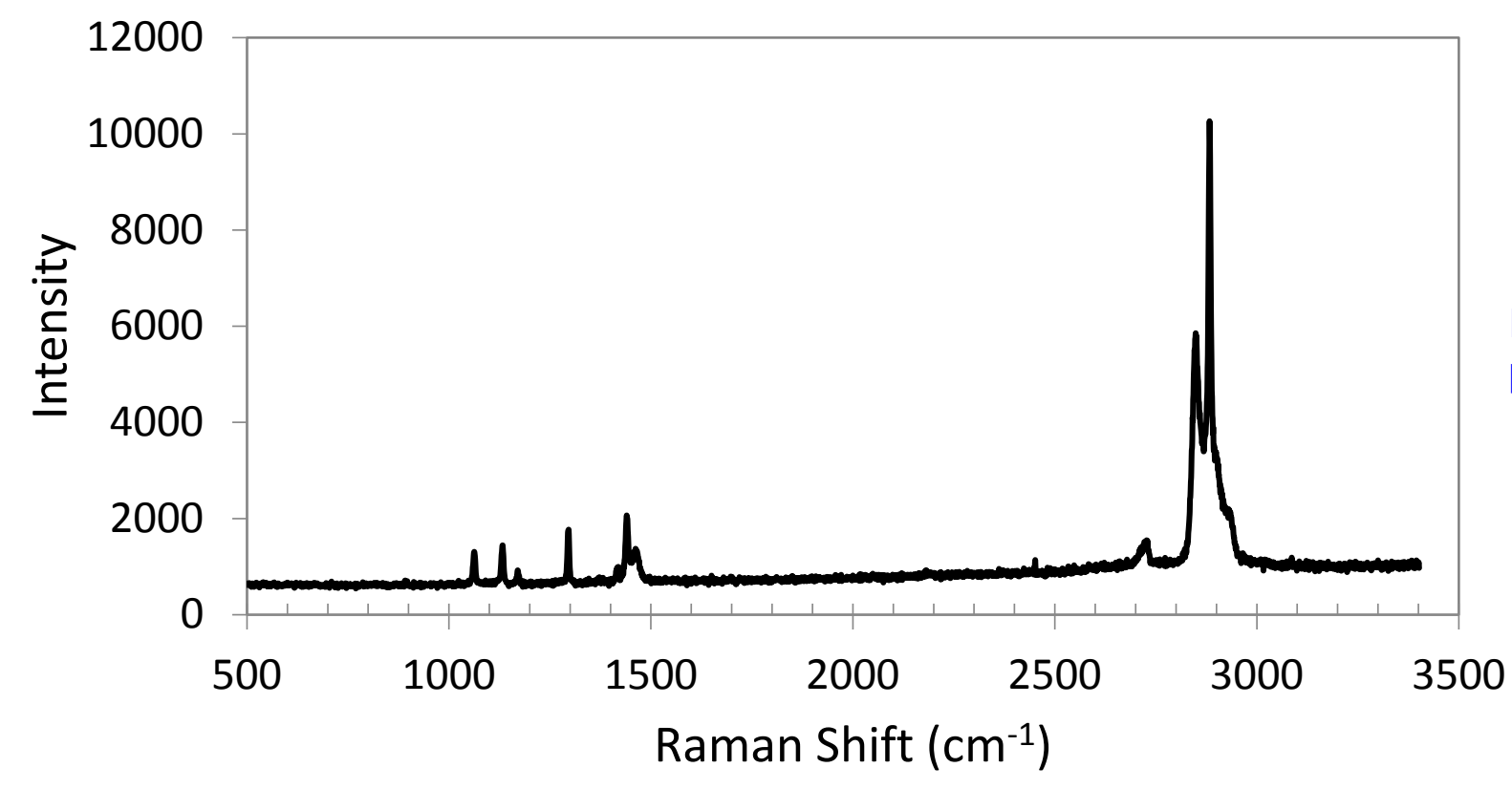

Figure 2b. MicroRaman spectrum of $\mathrm{C}_{40}$ particles

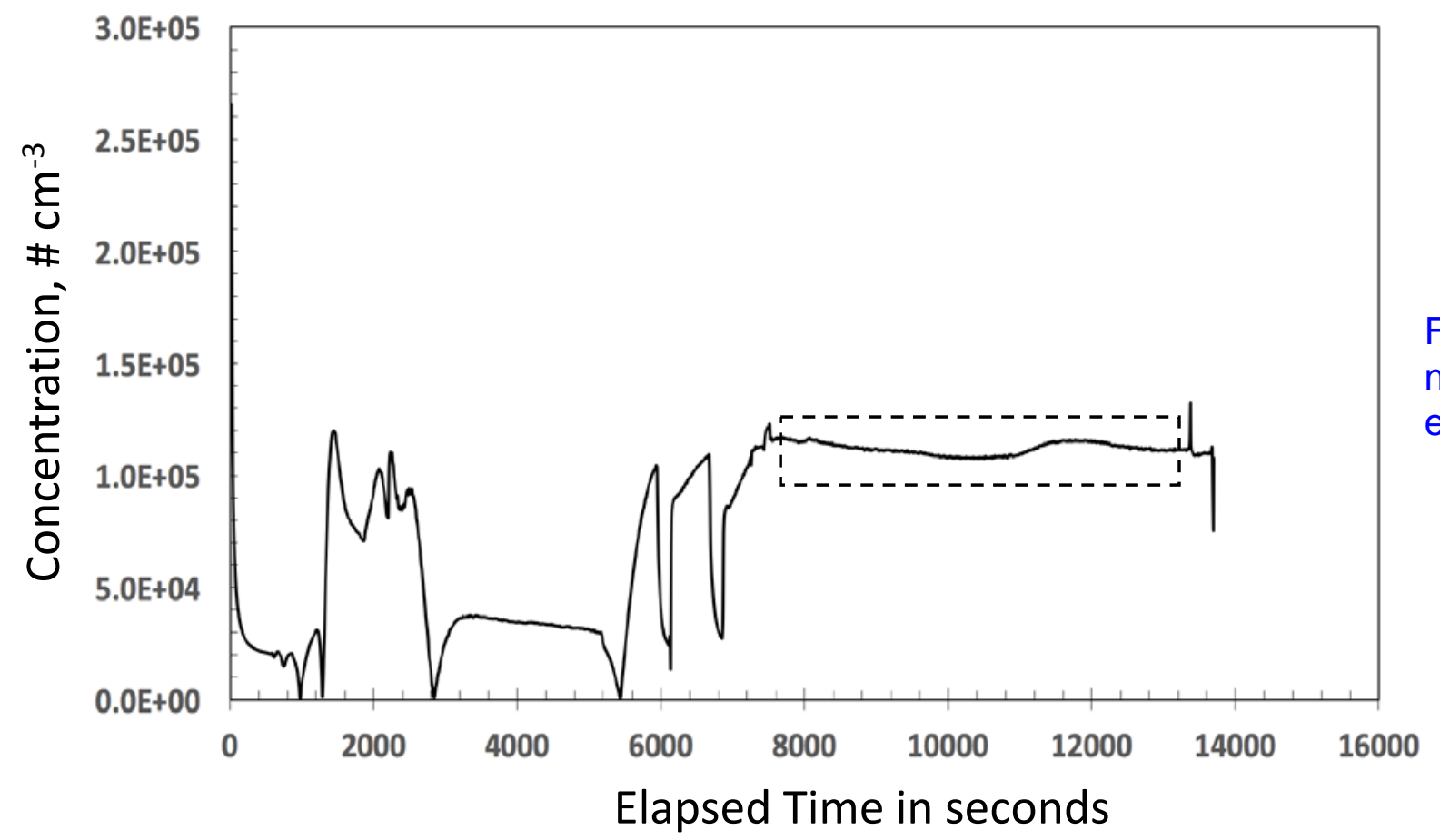

Figure 2c. Time trace of $\mathrm{C}_{40}$ particle number concentration. Dashed rectangle encompasses the experiment interval. 


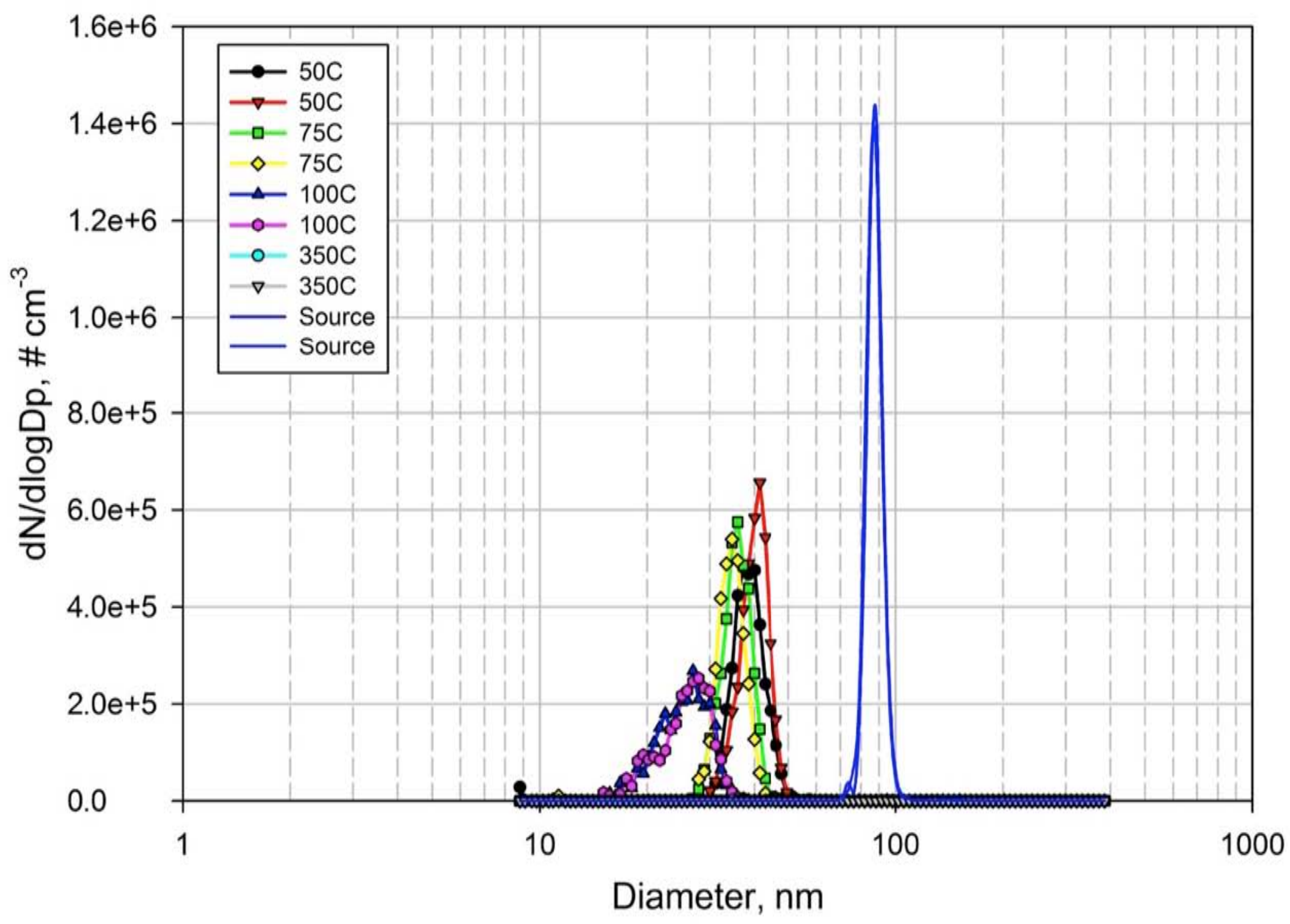

Figure 3. Measured thermogram of C40 test particles 


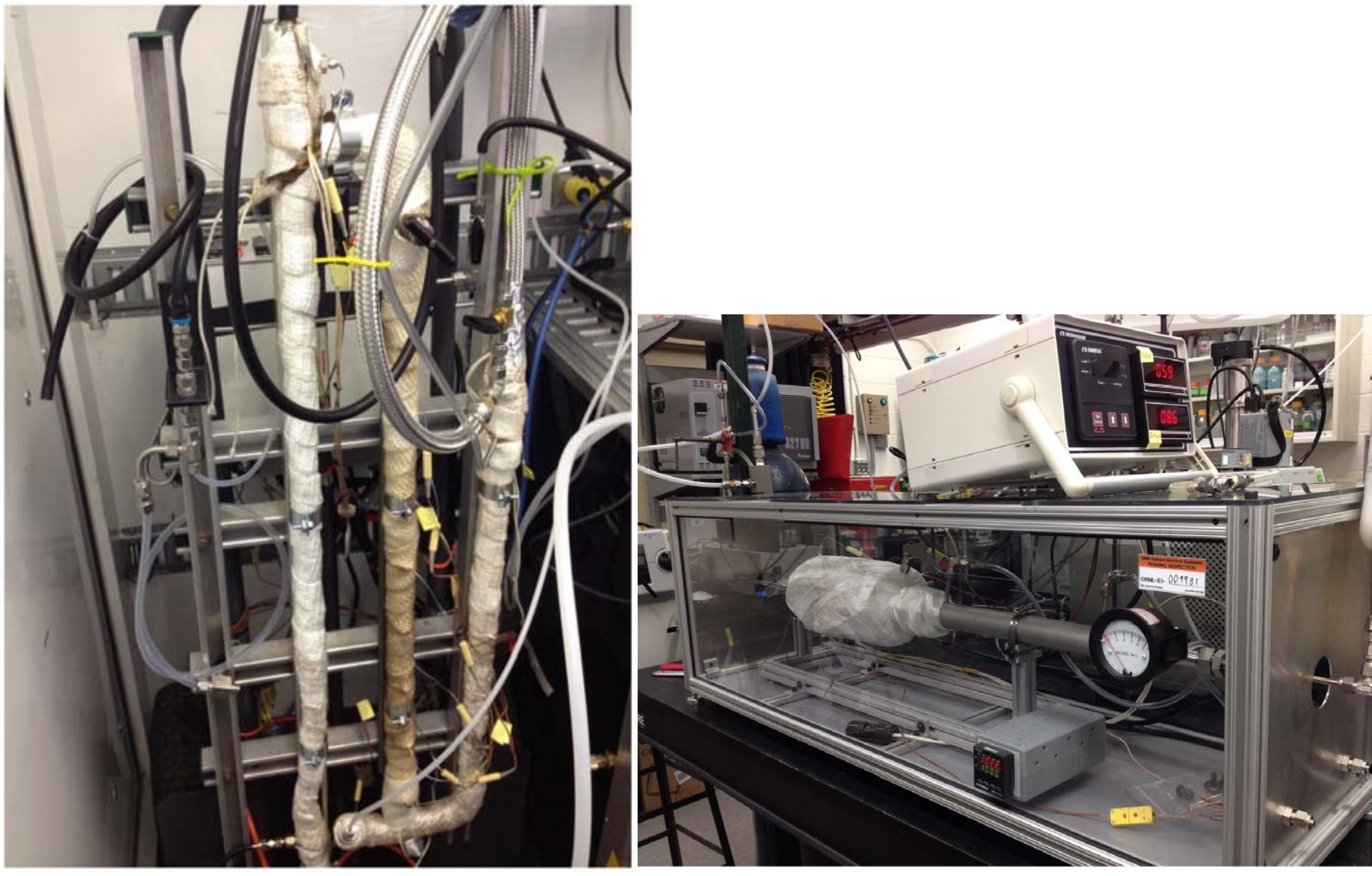

Figure 4a. ORNL PMP VPR tested on C-17 aircraft

Figure 4b. ORNL VPS. Version 3 tested on C-17 aircraft 

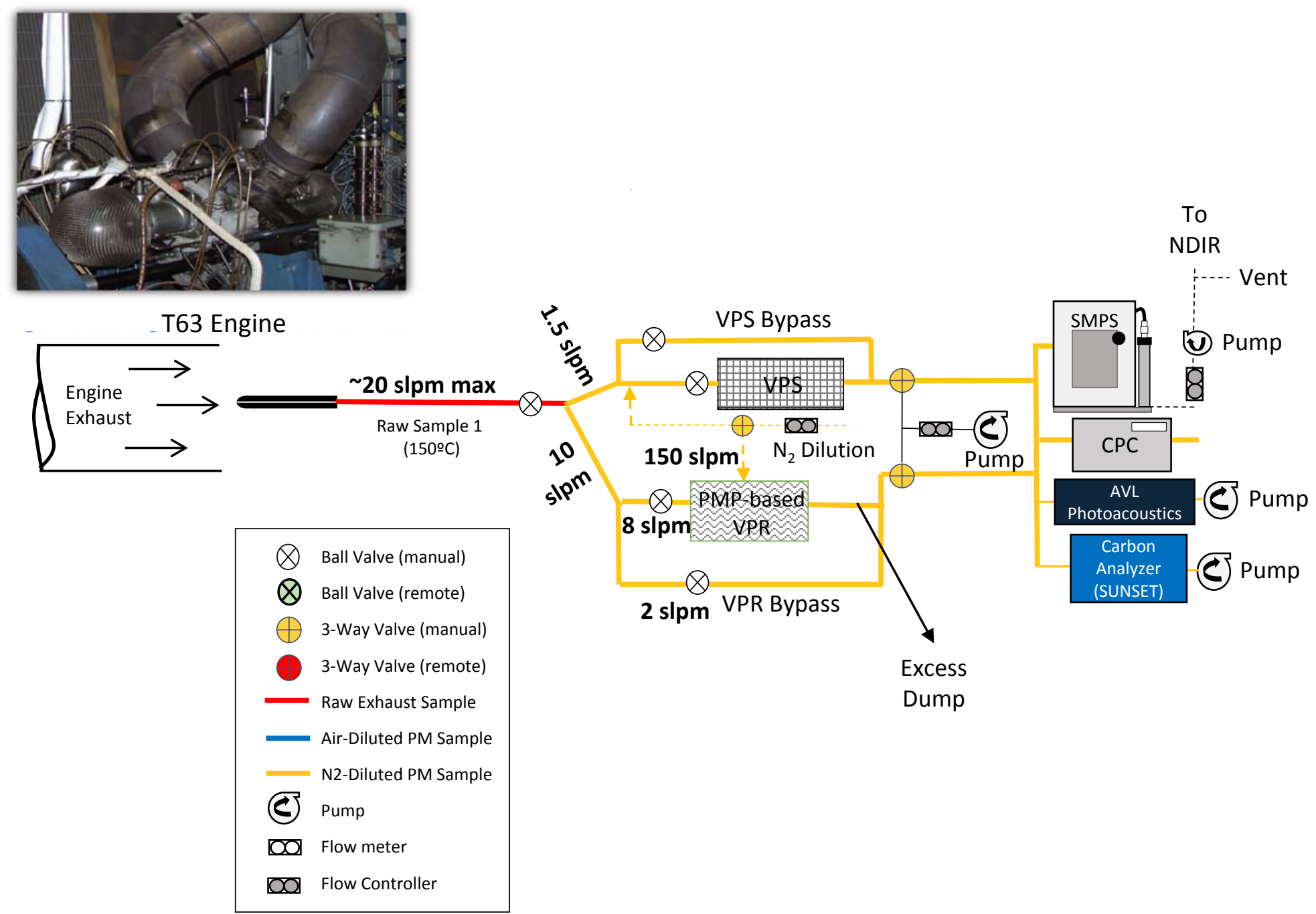

Figure 5. Schematic of field test on T63 engine installed on a test stand at WP AFRL 


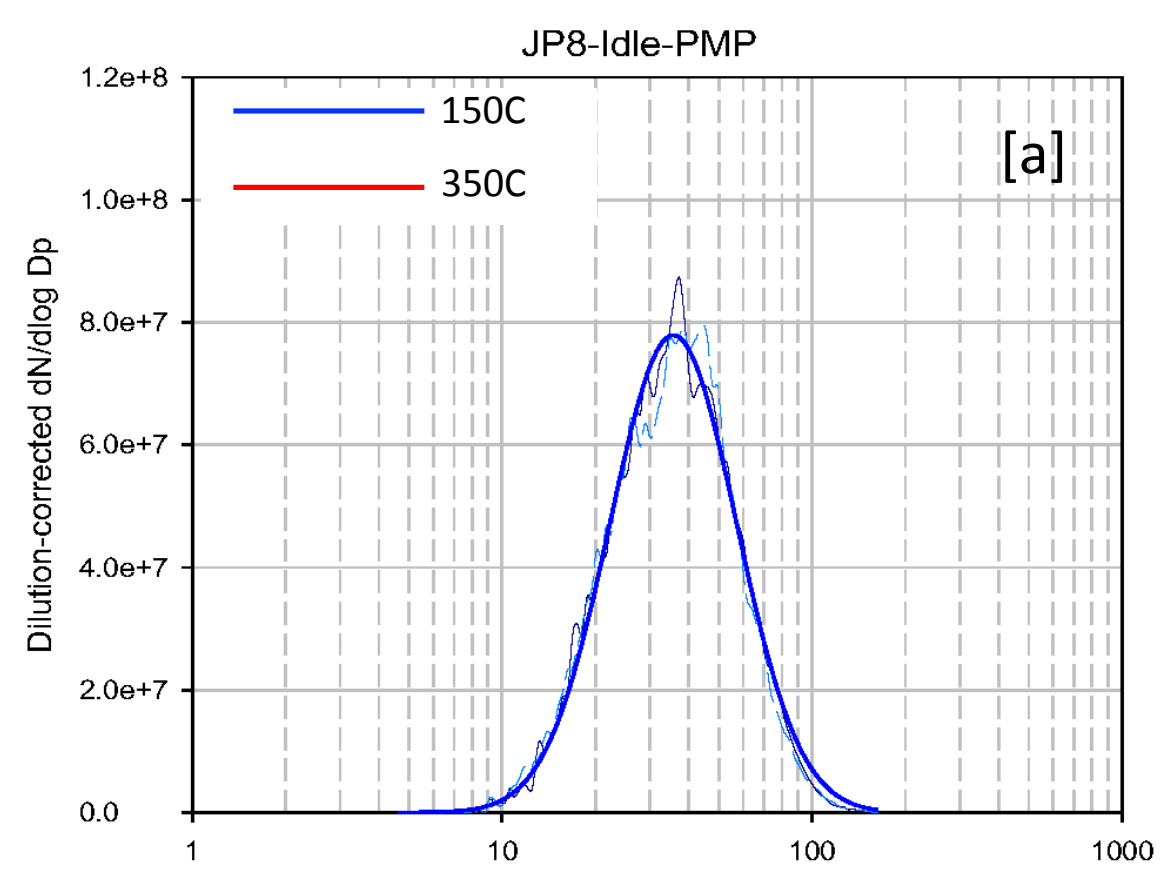

JP8-Cruise-PMP
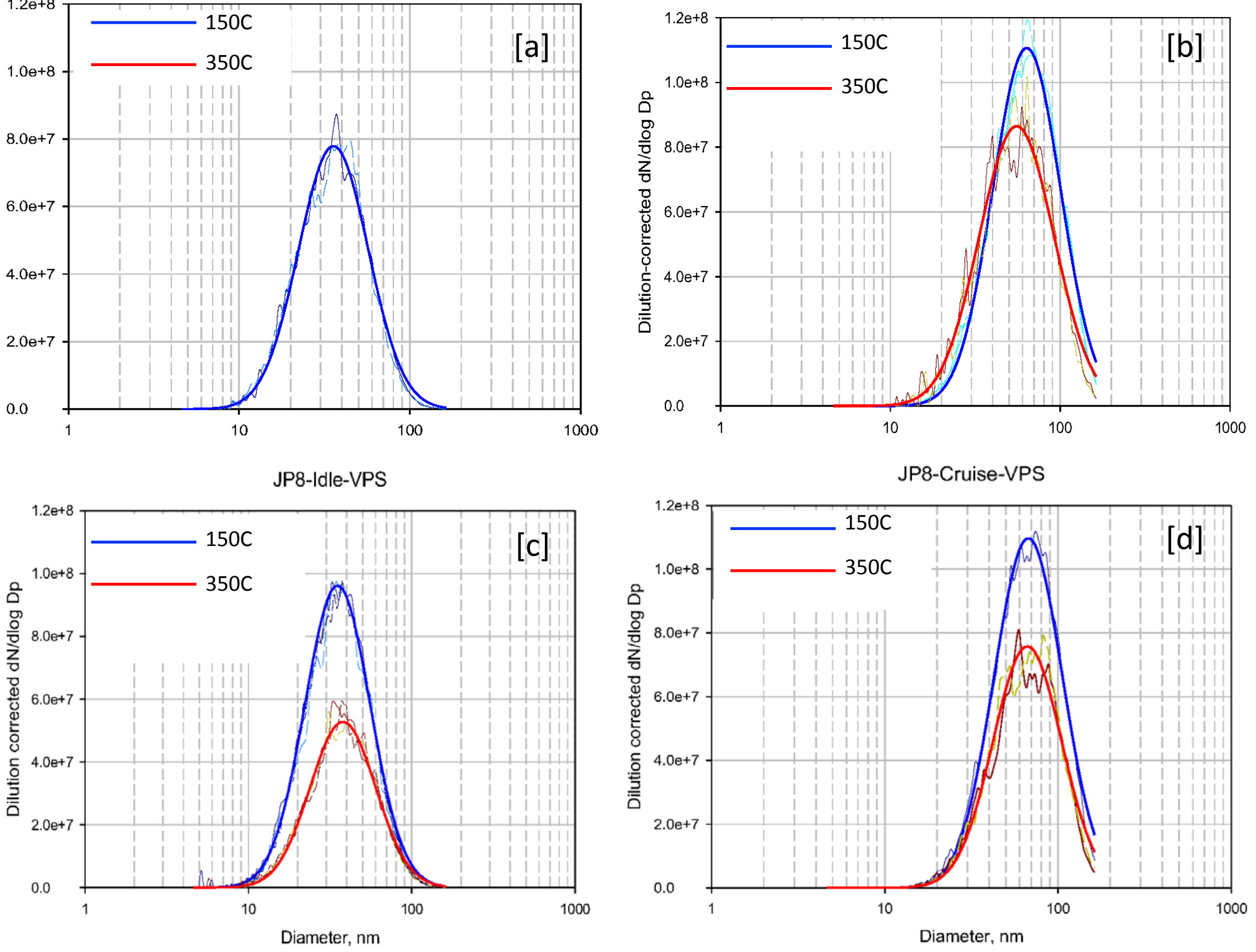

Figure 6. T63 engine test results 
JP8+high Aromatics-Cruise
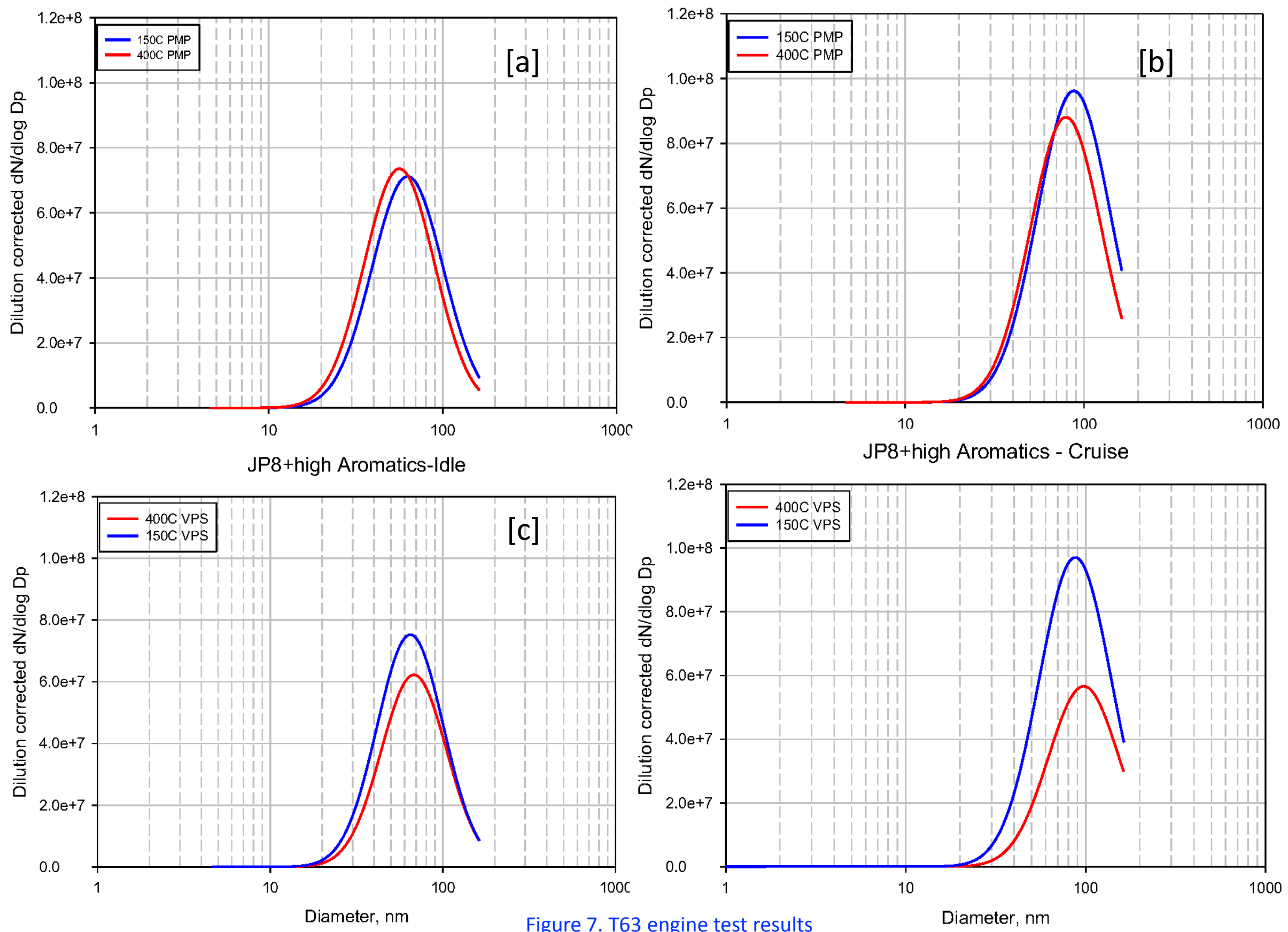


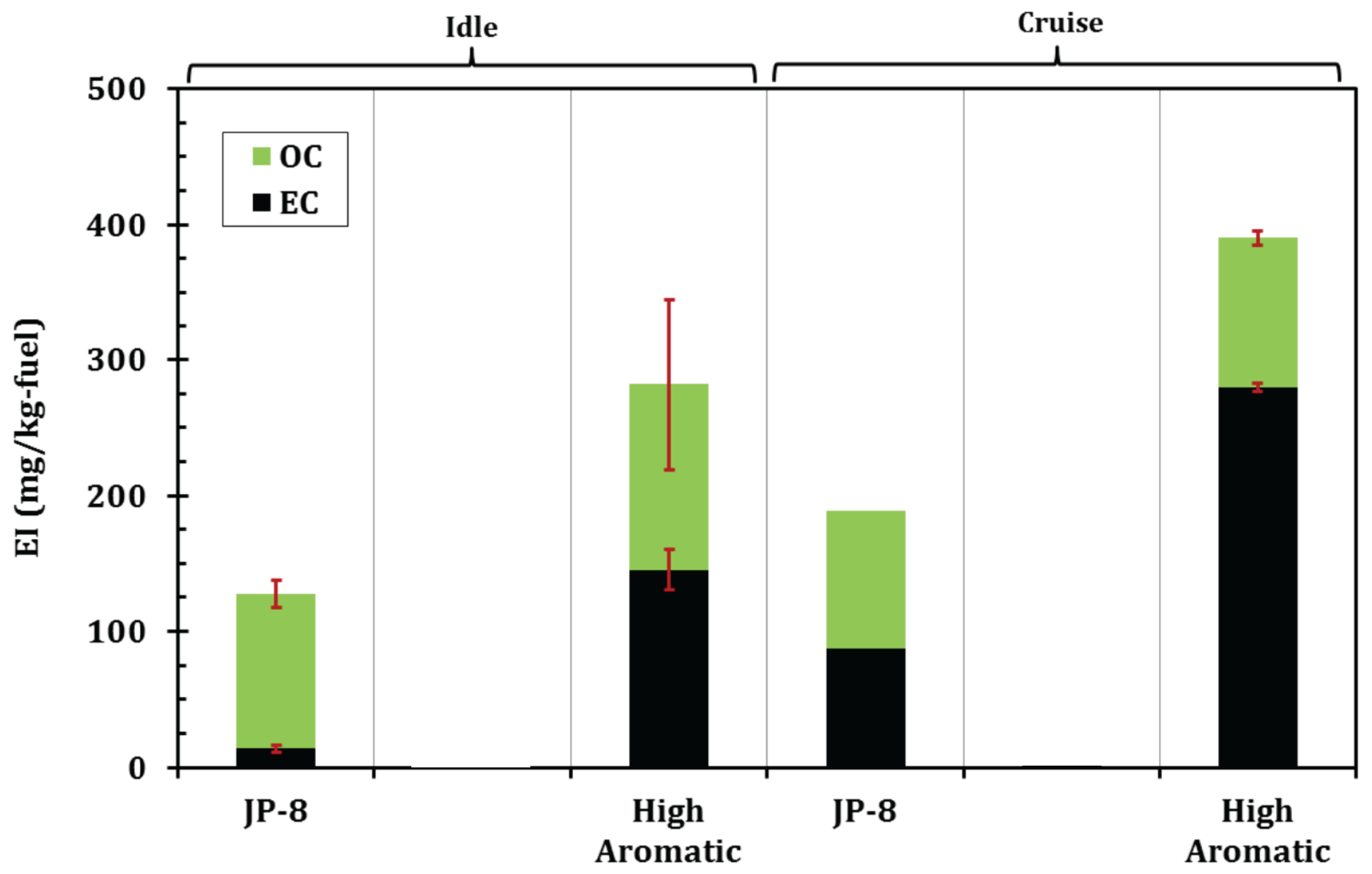

Figure 8. EC and OC contents of measured T63 samples 


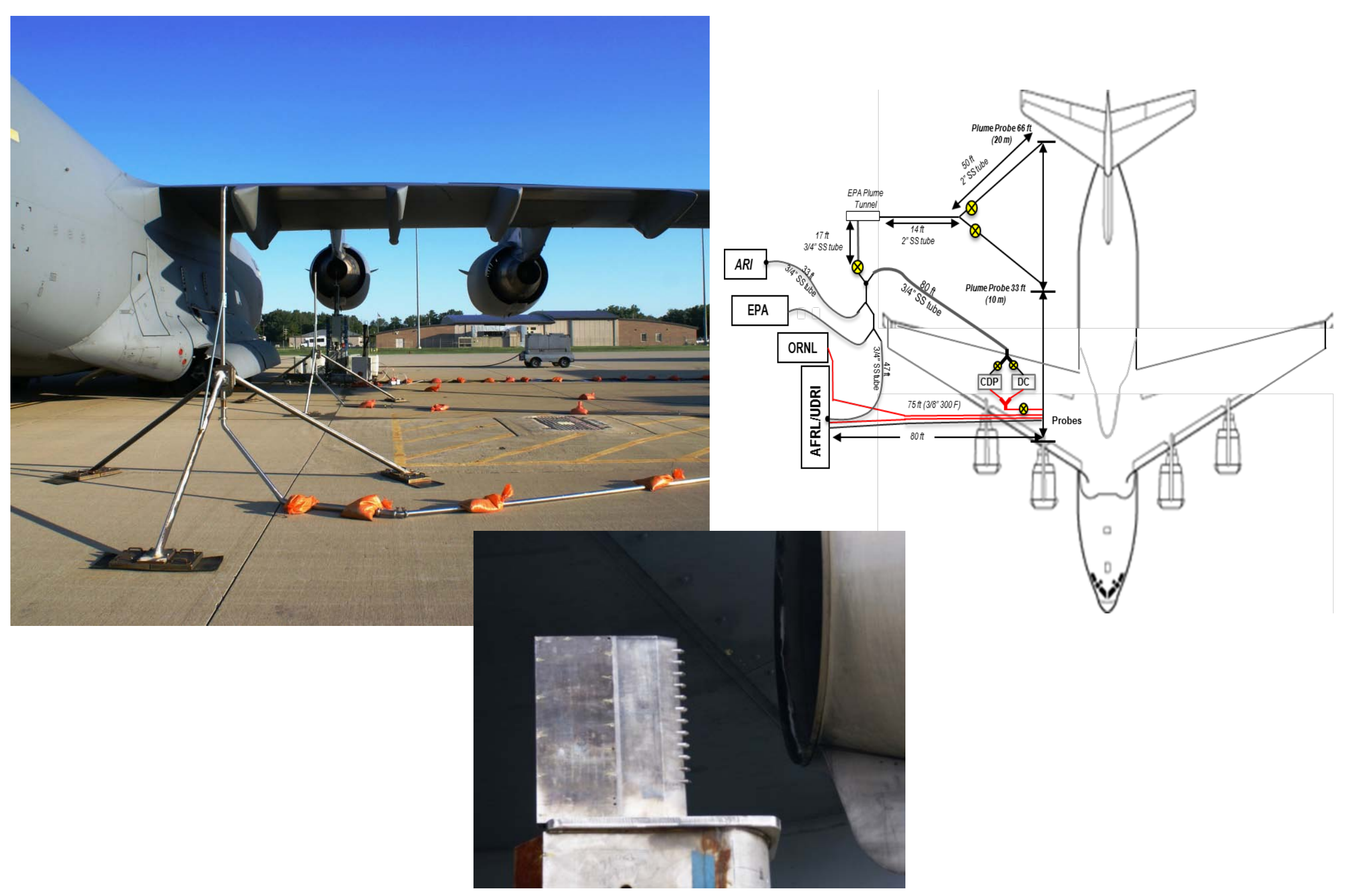

Figure 9. Sampled PW F117 engine on a C-17 aircraft parked at WPAFB 


\section{$\mathrm{C} 17-4 \%$}

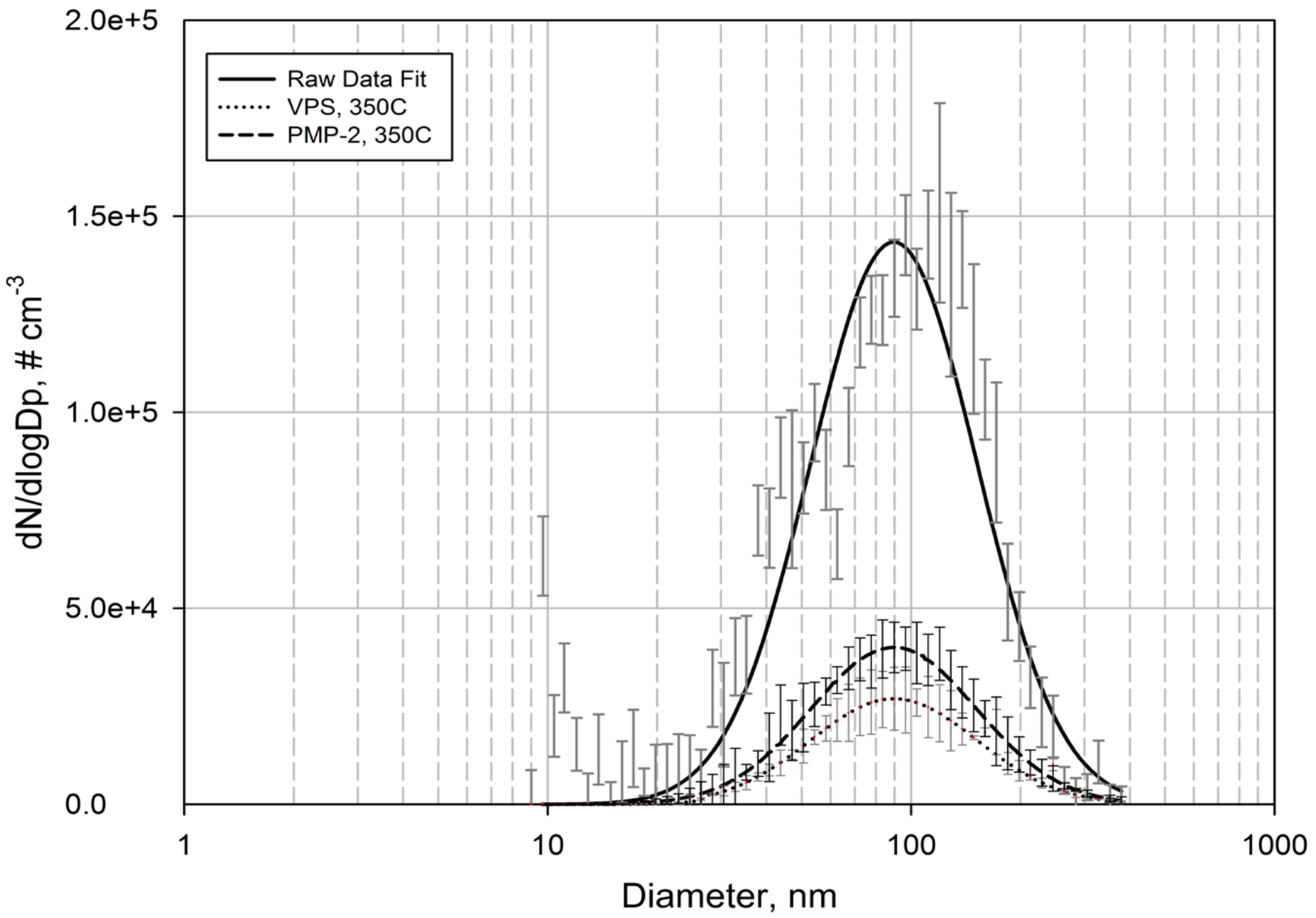

Figure 10. Lognormally-fitted particle size distributions for emissions from the C-17 engine operated at $4 \%$ rated thrust. Error bars represent the ranges of particle counts of the repeated scans. 


\section{C17-33\%}

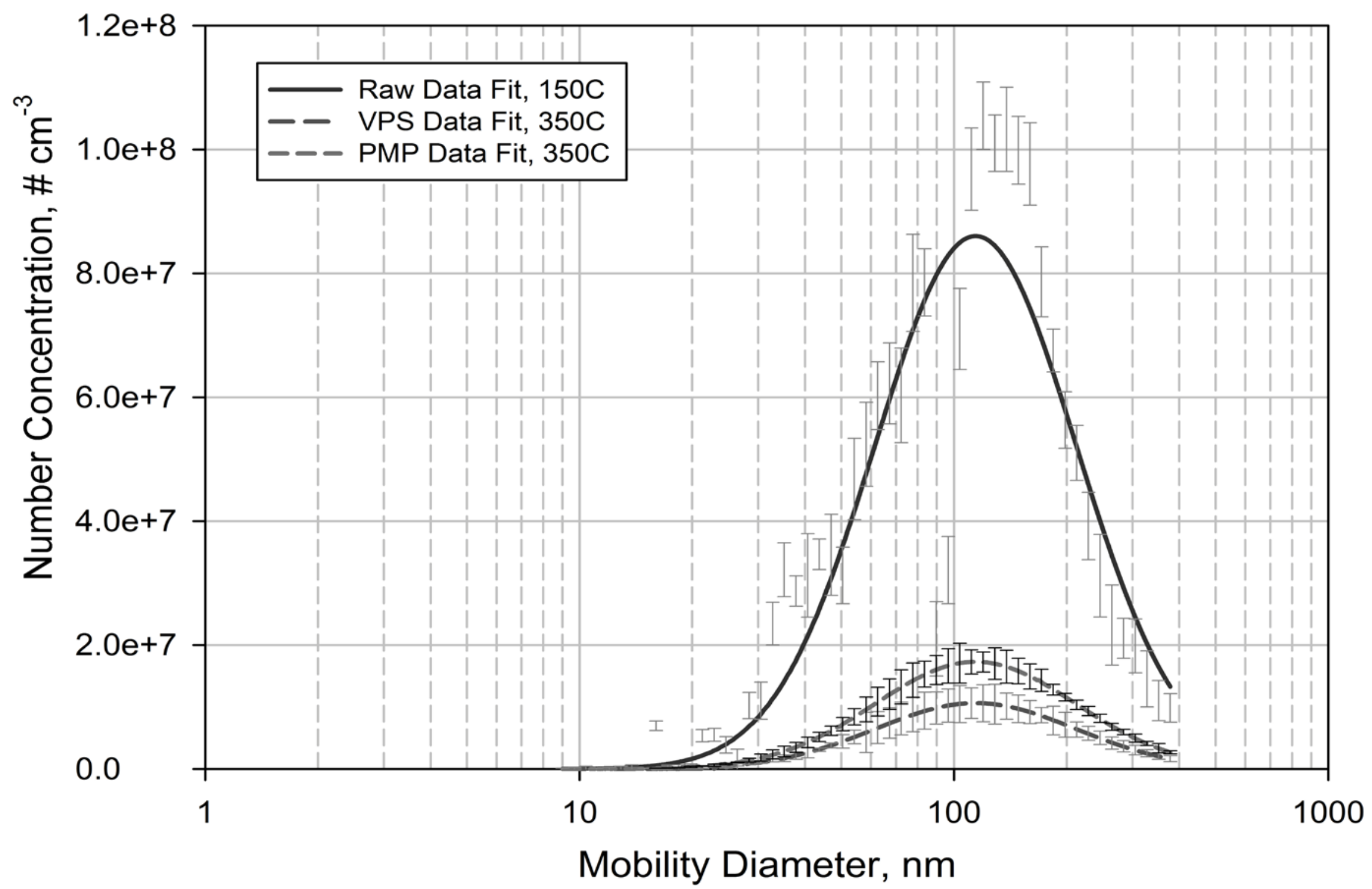

Figure 11. Lognormally-fitted particle size distributions for emissions from the C-17 engine operated at $33 \%$ rated thrust. Error bars represent the ranges of particle counts of the repeated scans. 